Pacific Journal of Mathematics

ON THE MEASURABILITY OF CONDITIONAL 


\title{
ON THE MEASURABILITY OF CONDITIONAL EXPECTATIONS
}

\author{
Albrecht IrLe
}

\begin{abstract}
It is shown that for a measurable stochastic process $V$ and a nondecreasing family of $\sigma$-algebras $\mathscr{A}_{t}$ there exists a measurable stochastic process $V^{*}$ such that $V^{*}(t, \cdot)$ is a version of $E\left(V(t, \cdot) \mid \mathscr{A}_{t}\right)$ for all $t$.
\end{abstract}

Let $(\Omega, \mathscr{A}, P)$ be a probability space (not necessarily complete), $T$ an interval (bounded or unbounded) of the real line and $V$ a real-valued stochastic process defined on $T \times \Omega$ which is a measurable process, see Doob [3, p.60]. Let $\mathscr{A}_{t}, t \in T, \mathscr{A}_{t} \subset \mathscr{A}$ form a nondecreasing family of $\sigma$-algebras. We shall prove in this note that under some boundedness condition on $V$ the conditional expectations with respect to $P, E\left(V(t, \cdot) \mid \mathscr{A}_{t}\right)$ can be chosen as to define a measurable process on $T \times \Omega$. A similar statement appears in a paper by Brooks [1] but there it is additionally assumed that the family of $\sigma$-algebras is left-continuous, and the proof given there does not seem to carry over to a general nondecreasing family.

Theorem. Suppose for each $t \in T: V(t, \cdot) \geqq 0 \quad P$-a.s. or $\int|V(t, \cdot)| d P<\infty$. Then there exists a measurable process $V^{*}$ such that for each $t \in T, V^{*}(t, \cdot)$ is a version of $E\left(V(t, \cdot) \mid \mathscr{A}_{t}\right)$.

Proof. Since for any $t \in T$

$$
E\left(V(t, \cdot) \mid \mathscr{A}_{t}\right)=E\left(V(t, \cdot)^{+} \mid \mathscr{A}_{t}\right)-E\left(V(t, \cdot)^{-} \mid \mathscr{A}_{t}\right)
$$

we may assume without loss of generality that for each $t \in T$ $V(t, \cdot) \geqq 0 \quad P$-a.s. Using the linearity and monotone convergence property of conditional expectations the theorem now is easily reduced to the case that $V$ is the characteristic function $I_{D}$ of some subset $D=B \times A$ of $T \times \Omega$ with $A \in \mathscr{A}$ and $B$ belonging to the Borel sets of $T$.

Since $E\left(I_{D}(t, \cdot) \mid \mathscr{A}_{t}\right)=I_{B}(t) E\left(I_{A} \mid \mathscr{A}_{t}\right)$ holds it is enough to show that $E\left(I_{A} \mid \mathscr{A}_{t}\right)$ can be chosen to form a measurable process. Let $\mathcal{M}$ denote the set of all random variables on $(\Omega, \mathscr{A}, P)$ taking values in $[0,1]$ with random variables that are equal $P$-a.e. identified. Then $\mathcal{M}$ is a metrizable topological space under the topology of convergence in 
probability. By Theorem 3 in Cohn [2] it is now sufficient to show that the mapping $E_{A}: T \rightarrow M$ with $E_{A}(t)=E\left(I_{A} \mid \mathscr{A}_{t}\right)$ has separable range and is measurable with respect to the Borel sets of M. $E\left(I_{A} \mid \mathscr{A}_{t}\right), t \in T$, forms a uniformly integrable martingale and so it follows from Theorem 11.2 in Doob [3], p. 358, that $E_{A}$ is continuous at all but countably many points of $T$. This yields at once that $E_{A}$ is measurable and furthermore - since $T$ is separable - that the range of $E_{A}$ is separable. This concludes the proof.

If the condition ' $V(t, \cdot) \geqq 0$ P-a.s. or $\int|V(t, \cdot)| d P<\infty$ ' is only required to hold for $\mu-a . a$. $t \in T, \mu$ being any measure on the Borel sets of $T$, then obviously there exists a measurable process $V^{*}$ which is a version of $E\left(V(t, \cdot) \mid \mathscr{A}_{t}\right)$ for $\mu$-a.a. $t \in T$.

\section{REFERENCES}

1. R. A. Brooks, Conditional expectations associated with stochastic processes, Pacific J. Math., 41 (1972), 33-42.

2. D. L. Cohn, Measurable choice of limit points and the existence of separable and measurable processes, Z. Wahrscheinlichkeitstheorie und Verw. Gebiete., 22 (1972), 161-165.

3. J. L. Doob, Stochastic Processes, New York, Wiley, 1953.

Received February 25, 1975 and in revised form February 22, 1977.

InStitut für Mathematische Statistik der Üniversität MünSter, Roxeler STR. 64, West Germany 



\section{Pacific Journal of Mathematics \\ Vol. 70, No. $1 \quad$ September, 1977}

William H. Barker, Noether's theorem for plane domains with hyperelliptic

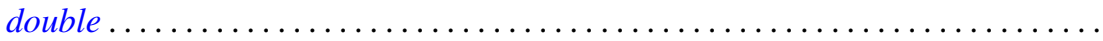

Michael James Beeson, Non-continuous dependence of surfaces of least area on the boundary curve ...................................... 11

Horst Behncke, Functions acting in weighted Orlicz algebras . . . . . . . . . . . . 19

Howard Edwin Bell, A commutativity study for periodic rings . . . . . . . . . . . 29

Peter Botta and Stephen J. Pierce, The preservers of any orthogonal group ....... 37

Douglas S. Bridges, The constructive Radon-Nikodým theorem ............. 51

James Dennis Brom, The theory of almost periodic functions in constructive

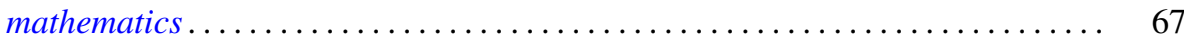

N. Burgoyne and C. Williamson, Semi-simple classes in Chevalley type groups ....

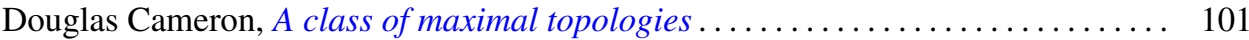

L. Carlitz, Enumeration of doubly up-down permutations . . . . . . . . . . . . . . 105

Paul Robert Chernoff, The quantum n-body problem and a theorem of

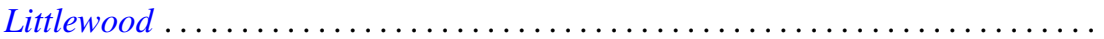

Jo-Ann Deborah Cohen, Locally bounded topologies on $F(X) \ldots \ldots \ldots \ldots \ldots \ldots$

Heinz Otto Cordes and Robert Colman McOwen, Remarks on singular elliptic

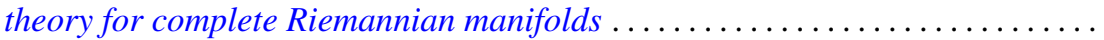

Micheal Neal Dyer, Correction to: "Rational homology and Whitehead

products"

Robert Fernholz, Factorization of Radonifying transformations

Lawrence Arthur Fialkow, A note on quasisimilarity. II ...... . .

Harvey Charles Greenwald, Lipschitz spaces of distributions on the surface of unit

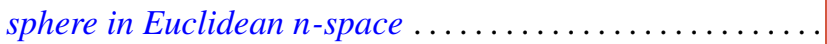

Albrecht Irle, On the measurability of conditional expectations

Tom (Roy Thomas Jr.) Jacob, Matrix transformations involving simple sequence

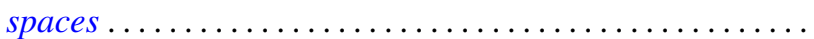

A. Katsaras, Continuous linear maps positive on increasing continuous

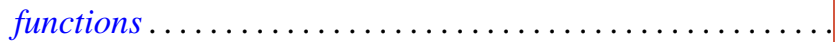

Kenneth Kunen and Judith Roitman, Attaining the spread at cardinals of cofinality

Lawrence Louis Larmore and Robert David Rigdon, Enumerating normal bundles

of immersions and embeddings of projective spaces ...... . .

Ch. G. Philos and V. A. Staïkos, Asymptotic properties of nonoscillatory solutions of differential equations with deviating argument .

Peter Michael Rosenthal and Ahmed Ramzy Sourour, On operator algebras containing cyclic Boolean algebras...

Polychronis Strantzalos, Strikt fast gleichgradig-stetige und eigentliche

Aktionen ...

Glenn Francis Webb, Exponential representation of solutions to an abstract

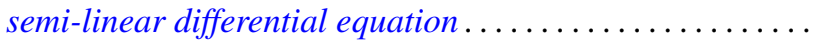

